\title{
Establishment and Epitope Mapping of Anti-Diacylglycerol Kinase $\alpha$ Monoclonal Antibody DaMab-8 for Immunohistochemical Analyses
}

\author{
Masato Sano, ${ }^{1}$ Mika K. Kaneko, ${ }^{1}$ Hiroyoshi Suzuki, ${ }^{2}$ and Yukinari Kato ${ }^{1,3}$
}

Diacylglycerol kinase (DGK) converts diacylglycerol (DG) into phosphatidic acid (PA). DGK $\alpha, 1$ of the 10 DGK isozymes, is involved in $\mathrm{T}$ cell function. In the present study, we describe a specific monoclonal antibody DaMab-8 (mouse $\mathrm{IgG}_{1}$, kappa) against $\mathrm{DGK} \alpha$, which is extremely useful for performing immunohistochemical analysis for $\mathrm{T}$ cells in oropharyngeal squamous cell carcinomas. Furthermore, we characterized the binding epitope of DaMab-8 using Western blotting and found that the sites Asn610, Leu611, Trp612, Gly613, Asp614, His619, Tyr623, and Gly624 of DGK $\alpha$ are important for facilitating the DaMab-8 binding to the DGK $\alpha$ protein. Thus, DaMab-8 could be advantageous for immunohistochemical analyses toward clarifying the distribution of DGK $\alpha$-expressing $\mathrm{T}$ cells in every pathophysiological tissue.

Keywords: diacylglycerol kinase, DGK $\alpha$, monoclonal antibody, epitope mapping

\section{Introduction}

D IACYLGLYCEROL KINASE (DGK) phosphorylates diacylglycerol (DG) to produce phosphatidic acid (PA). ${ }^{(1,2)}$ DG is a neutral lipid derived from various sources, including phosphatidylinositol 4,5-bisphosphate and phosphatidylcholine, and it serves as a second messenger that activates the conventional and novel types of the protein kinase C (PKC) family, RasGRP, Unc-13, and canonical transient receptor potential channels. ${ }^{(2,3)} \mathrm{PA}$ functions as a messenger molecule that activates the hypoxia-inducible factor (HIF)- $1 \alpha$, atypical PKC $\zeta$, and mammalian target of rapamycin. DGK constitutes an enzyme family comprising 10 isozymes of the mammalian species. $^{(1,2)}$ Each isozyme possesses a distinct molecular structure and a subcellular localization pattern. DGK $\alpha$ is the first identified enzyme of $80-\mathrm{kDa}$ size that contains an EF-hand motif ( $\mathrm{Ca}^{2+}$-binding site), a $\mathrm{Zn}$ finger (C1 domain, DG-binding site), and a catalytic domain. DGK $\alpha$ regulates cell proliferation in response to IL-2 stimulation in T cells ${ }^{(3)}$ and is involved in $\mathrm{T}$ cell receptor (TCR) signaling via the modulation of the RasGRP activity. ${ }^{(4)}$ T cells isolated from DGK $\alpha$-deficient mice demonstrate an altered activity of TCR signaling and hyperproliferation. ${ }^{(5)}$ DGK $\alpha$ is expressed in T lymphocytes abundantly, in which it facilitates the nonresponsive state known as clonal anergy. ${ }^{(5)}$ Anergy induction in T cells represents the main mechanism by which advanced tumors avoid immune action. ${ }^{(6)}$
Because only few specific anti-DGK $\alpha$ monoclonal antibodies (mAbs) are available to detect human DGK $\alpha$ using immunohistochemistry, the localization of DGK $\alpha$-expressing cells remains unclear. Recently, we have developed DaMab-2 (mouse $\mathrm{IgG}_{1}$, kappa), a specific mAb against DGK $\alpha .{ }^{(7)}$ DaMab-2 is extremely useful in immunocytochemical analysis using $\mathrm{HeLa}$ cells. We further characterized the binding epitope of DaMab-2 using Western blotting and revealed that the Cys246, Lys249, Pro252, and Cys 253 sites of DGK $\alpha$ are important for facilitating DaMab-2 binding to the DGK $\alpha$ protein. ${ }^{(8)}$ However, DaMab-2 was not applicable for immunohistochemical analysis.

In the present study, we report a novel anti-human DGK $\alpha$ mAb DaMab-8 (mouse IgG $_{1}$, kappa) that is extremely useful in immunohistochemical analysis. Furthermore, we have characterized the binding epitope of DaMab- 8 using Western blotting.

\section{Materials and Methods}

\section{Plasmid preparation}

Human DGK $\alpha$ cDNA $^{(9)}$ was synthesized and subcloned into the expression vector pMAL-c2 (New England Biolabs, Inc., Beverly, MA), along with PA tag (GVAMPGAEDDVV), ${ }^{(10)}$ using the In-Fusion HD Cloning Kit (Takara Bio, Inc., Shiga, Japan); the resultant construct was named pMAL-c2-DGK $\alpha$-PA. The deletion mutants of DGK $\alpha$

\footnotetext{
${ }^{1}$ Department of Antibody Drug Development, Tohoku University Graduate School of Medicine, Sendai, Japan.

${ }^{2}$ Department of Pathology and Laboratory Medicine, Sendai Medical Center, Sendai, Japan.

${ }^{3}$ New Industry Creation Hatchery Center, Tohoku University, Sendai, Japan.
}

(c) Masato Sano et al. 2019; Published by Mary Ann Liebert, Inc. This Open Access article is distributed under the terms of the Creative Commons License (http://creativecommons.org/licenses/by/4.0), which permits unrestricted use, distribution, and reproduction in any medium, provided the original work is properly cited. 
produced using PCR were subcloned into pMAL-c2 with PA tag using the In-Fusion PCR Cloning Kit. The substitution of DGK $\alpha$ amino acids 605-630 with either alanine or glycine in dN561 of DGK $\alpha$ was performed using the QuikChange Lightning Site-Directed Mutagenesis Kit (Agilent Technologies, Inc., Santa Clara, CA). These constructs were verified using direct DNA sequencing.

\section{Western blotting}

Competent Escherichia coli TOP-10 cells (Thermo Fisher Scientific, Inc., Waltham, MA) were transformed and cultured overnight at $37^{\circ} \mathrm{C}$ in Luria-Bertani medium (Thermo Fisher Scientific, Inc.,) containing $100 \mu \mathrm{g} / \mathrm{mL}$ ampicillin (FUJIFILM Wako Pure Chemical Corporation, Osaka, Japan). The cell pellets were resuspended in phosphate-buffered solution containing $1 \%$ Triton $\mathrm{X}-100$ and $50 \mu \mathrm{g} / \mathrm{mL}$ aprotinin (Sigma-Aldrich). Lysates were immunoprecipitated using amylose resin (New England Biolabs, Inc.) and boiled in sodium dodecyl sulfate (SDS) sample buffer (Nacalai Tesque, Inc., Kyoto, Japan). The samples were electrophoresed on $5 \%-20 \%$ polyacrylamide gels (FUJIFILM Wako Pure Chemical Corporation) and transferred onto a polyvinylidene difluoride membrane (Merck KGaA, Darmstadt, Germany). After blocking with 4\% skim milk (Nacalai Tesque, Inc.) for
1 hour, the membrane was incubated with DaMab-8 for 1 hour, followed by peroxidase-conjugated anti-mouse IgG (1:2000 dilution; Agilent Technologies, Inc.) for 1 hour. The membrane was also incubated with NZ-1 (anti-PA tag) for 1 hour, followed by biotin-conjugated anti-rat IgG (1:1000 dilution; Agilent Technologies, Inc.) for 30 minutes, and further incubated with the avidin-biotin complex (Vector laboratories, Inc., Burlingame, CA) for 30 minutes. The membrane was finally developed with the ImmunoStar LD Chemiluminescence Reagent (FUJIFILM Wako Pure Chemical Corporation) using the Sayaca-Imager (DRC Co., Ltd., Tokyo, Japan). All procedures of Western blotting were performed at room temperature.

\section{Immunohistochemical analyses}

Our study examined a patient with oropharyngeal squamous cell carcinoma who underwent surgery at the Sendai Medical Center. Informed consent for sample procurement and subsequent data analyses was obtained from the patient or the patient's guardian. The tissue samples were processed to produce $4-\mu \mathrm{m}$ paraffin-embedded tissue sections that were directly autoclaved in citrate buffer ( $\mathrm{pH}$ 6.0; Nichirei Biosciences, Inc., Tokyo, Japan) for 20 minutes and blocked using the SuperBlock T20 (PBS) Blocking Buffer (Thermo
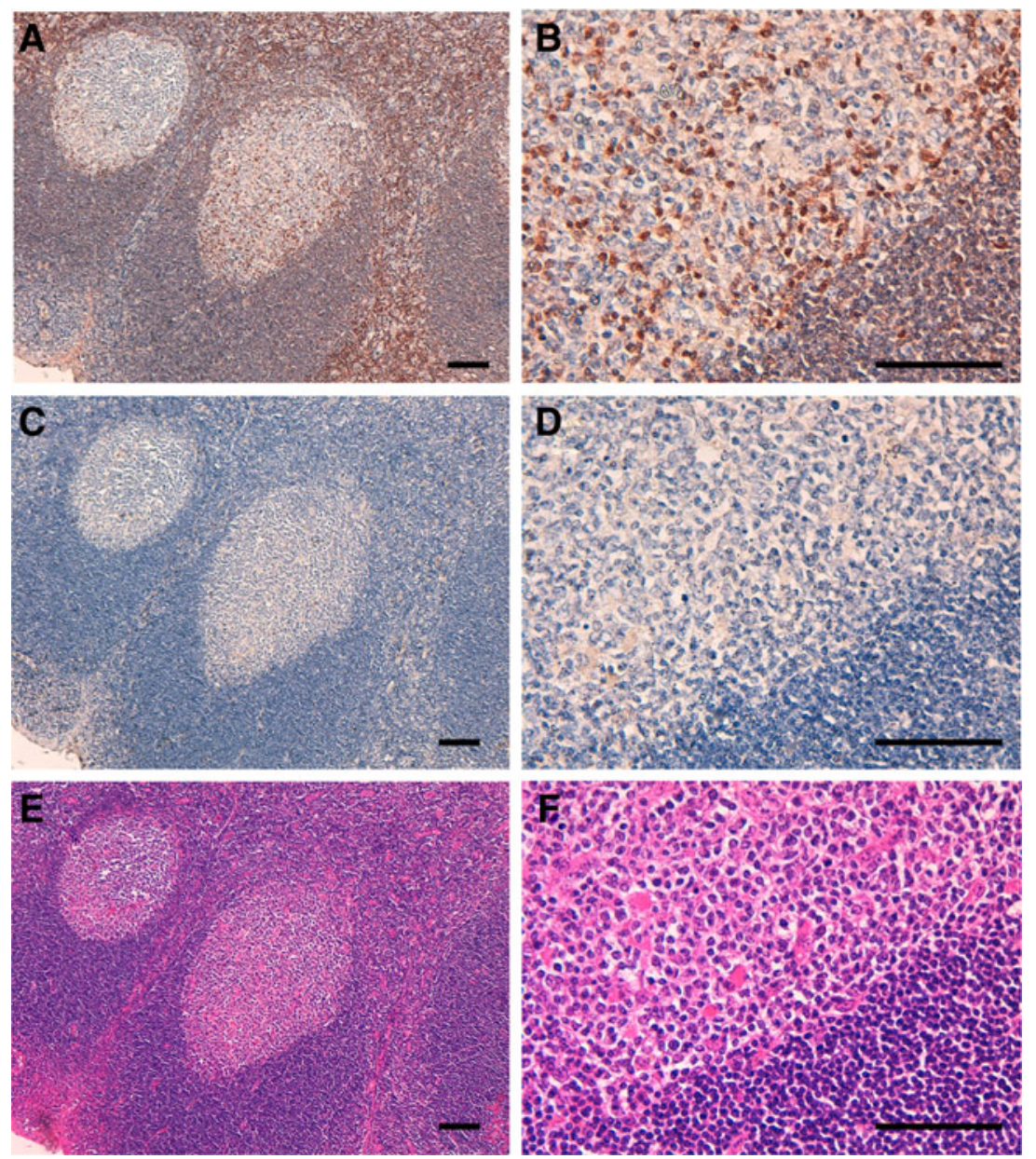

FIG. 1. Immunohistochemical analysis using DaMab-8 against oropharyngeal squamous cell carcinomas. Tissue sections were incubated with DaMab-8 $(1 \mu \mathrm{g} / \mathrm{mL} ; \mathbf{A}, \mathbf{B})$ or blocking buffer $(\mathbf{C}, \mathbf{D})$ for 1 hour at room temperature and treated using the Envision Kit (Agilent Technologies, Inc.) for 30 minutes. Scale bar $=100 \mu \mathrm{m}$. (E, F) Hematoxylin and eosin staining. 
A

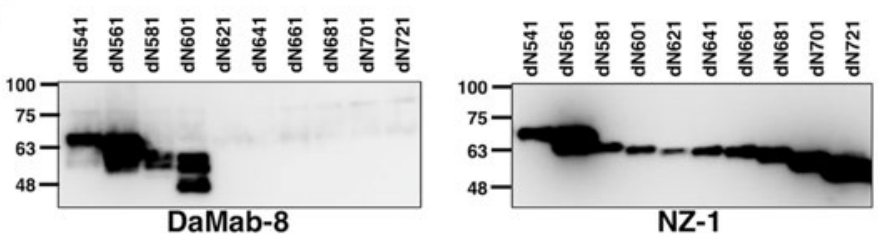

B

MBP-hDGK $\alpha-P A$ tag

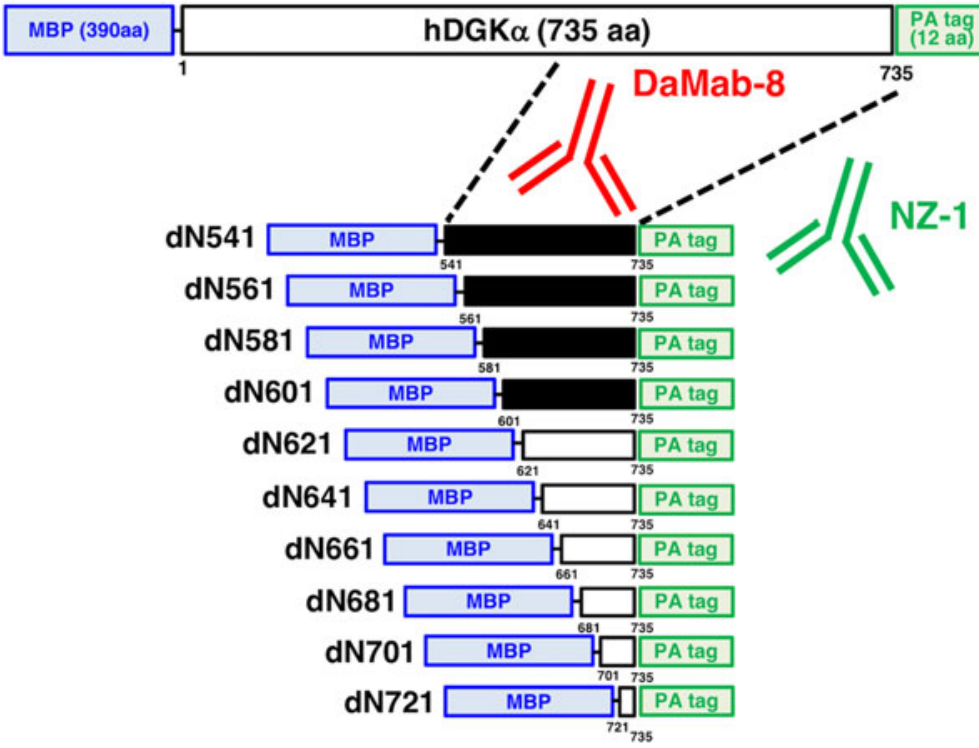

FIG. 2. Production of $D G K \alpha$ deletion mutants. (A) Immunoprecipitates of deletion mutants were electrophoresed and transferred onto PVDF membranes. After blocking, the membranes were incubated with $1 \mu \mathrm{g} / \mathrm{mL}$ of DaMab-8 or anti-PA tag (NZ-1), followed by incubation with peroxidase-conjugated anti-mouse or anti-rat IgG. (B) Schematic illustration of DaMab-8 epitope. Black bars, deletion mutants detected by DaMab-8; white bars, deletion mutants not detected by DaMab-8. hDGK $\alpha$, human diacylglycerol kinase $\alpha$; MBP, maltose-binding protein; PVDF, polyvinylidene difluoride.

A

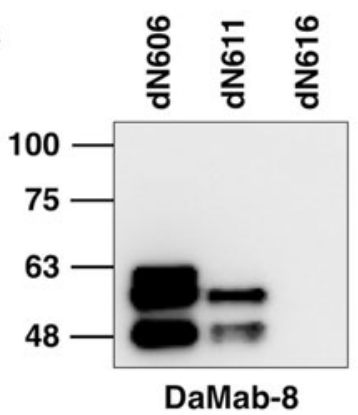

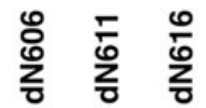

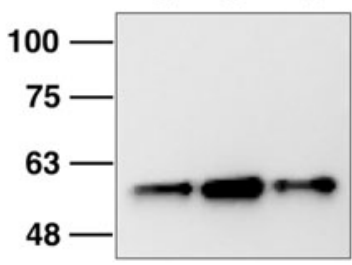

NZ-1

B MBP-hDGK $\alpha$-PA (immunogen)

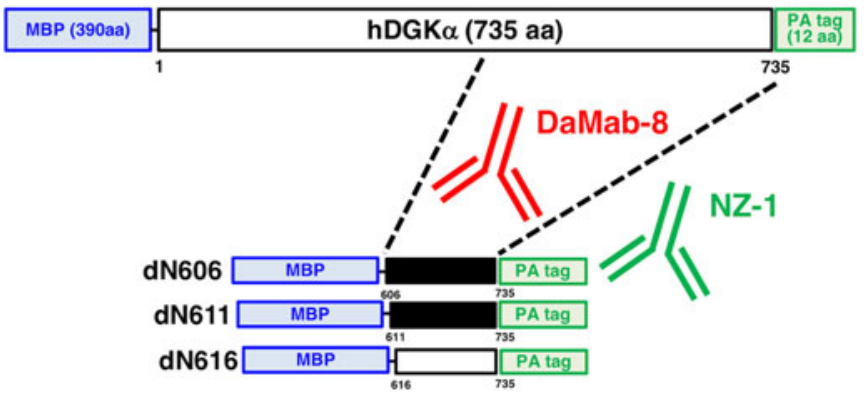

FIG. 3. Production of additional deletion mutants of DGK $\alpha$. (A) Immunoprecipitates of deletion mutants were electrophoresed and transferred onto PVDF membranes. After blocking, the membranes were incubated with $1 \mu \mathrm{g} / \mathrm{mL}$ of DaMab-8 and anti-PA tag (NZ-1), followed by incubation with peroxidase-conjugated anti-mouse IgG and biotin-conjugated anti-rat IgG, respectively. (B) Schematic illustration of DaMab-8 epitope. Black bars, deletion mutants detected by DaMab-8; white bars, deletion mutants not detected by DaMab-8. 
Fisher Scientific, Inc., $)$, incubated with DaMab-8 $(1 \mu \mathrm{g} / \mathrm{mL})$ for 1 hour at the room temperature, and then treated using the Envision Kit (Agilent Technologies, Inc.) for 30 minutes. The tissue sections were stained using 3, $3^{\prime}$-diaminobenzidine tetrahydrochloride (DAB; Agilent Technologies, Inc.) for 2 minutes, and counterstaining was performed using hematoxylin (FUJIFILM Wako Pure Chemical Corporation).

\section{Results and Discussion}

Several anti-DGK $\alpha$ mAbs are commercially available and are reportedly useful in Western blotting and for immunohistochemical analyses. ${ }^{(11)}$ Furthermore, we have developed DaMab-2 (mouse $\mathrm{IgG}_{1}$, kappa), a specific mAb against DGK $\alpha$, which is extremely useful in immunocytochemical analysis. ${ }^{(7)}$ The binding epitopes of DaMab-2 were determined to be Cys246, Lys249, Pro252, and Cys253 of DGK $\alpha{ }^{(8)}$ Unfortunately, DaMab-2 was not applicable for immunohisto- chemical analysis. We have previously immunized mice with recombinant DGK $\alpha$ and developed several anti-DGK clones. ${ }^{(7)}$ One of these clones, DaMab-8, recognized only $\mathrm{DGK} \alpha$ in ELISA and showed no reaction with other isozymes, such as DGK $\gamma, \mathrm{DGK} \zeta$, DGK $\eta$, and DGK $\delta$ (data not shown). DGK $\alpha$ was reported to be expressed in T lymphocytes abundantly, in which it facilitates the nonresponsive state known as clonal anergy. ${ }^{(5)}$ Immunohistochemical screening revealed that DaMab-8 was extremely useful in immunohistochemical analysis for $\mathrm{T}$ cells in oropharyngeal squamous cell carcinomas (Fig. 1).

We next moved on to the determination of the binding epitope of DaMab-8. As shown in Figure 2, we produced $10 \mathrm{~N}-$ terminal deletion mutants of $\mathrm{DGK} \alpha$ (i.e., dN541, dN561, dN581, dN601, dN621, dN641, dN661, dN681, dN701, and dN721). Western blotting demonstrated that DaMab-8 detected dN541, dN561, dN581, and dN601 but not dN621, dN641, dN661, dN681, dN701, and dN721, although all
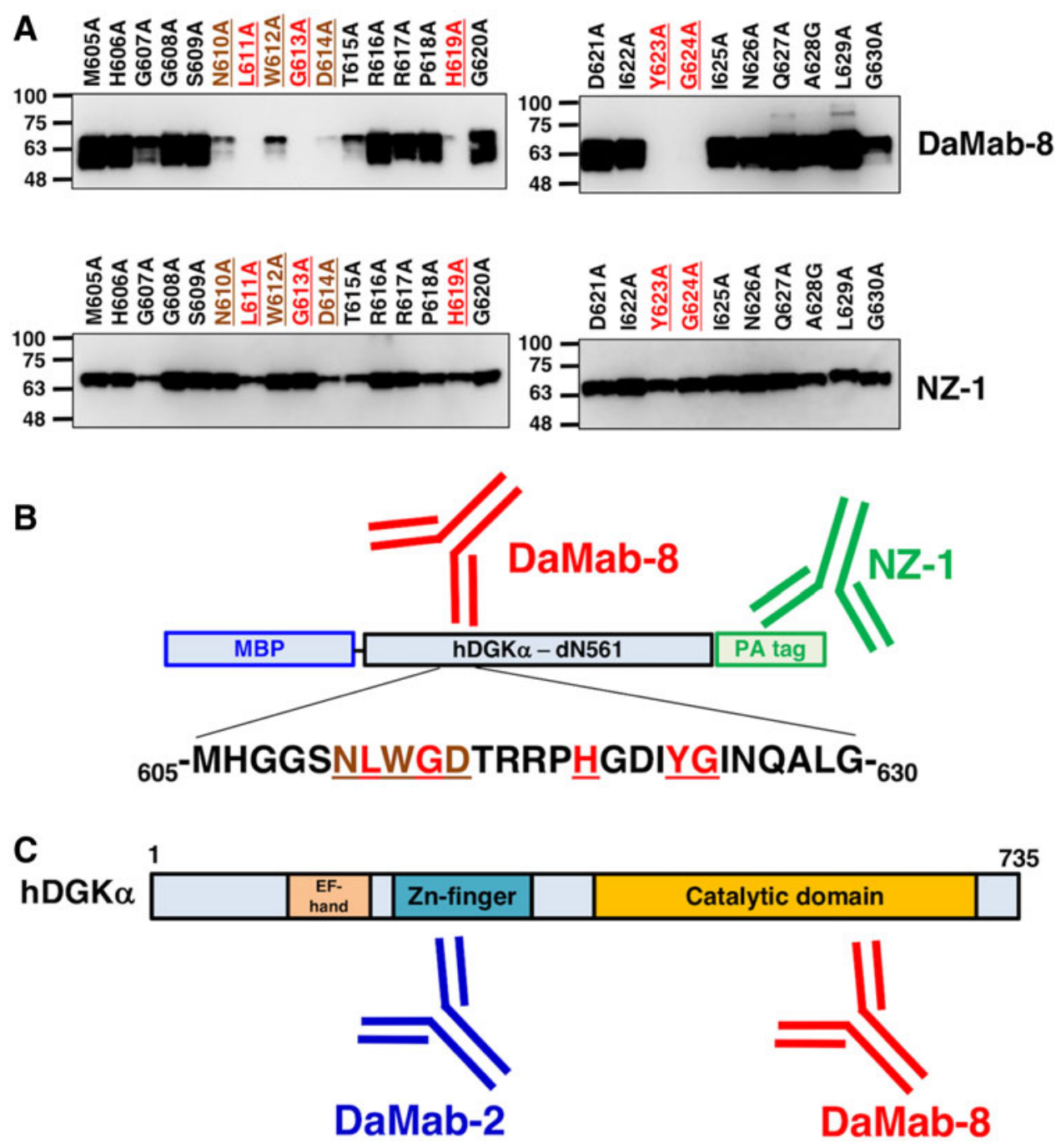

FIG. 4. Epitope mapping of DaMab-8 using point mutants of DGK $\alpha$. (A) Immunoprecipitates of point mutants were electrophoresed and transferred onto PVDF membranes. After blocking, the membranes were incubated with $1 \mu \mathrm{g} / \mathrm{mL}$ of DaMab-8 and anti-PA tag (NZ-1), followed by incubation with peroxidase-conjugated anti-mouse IgG and biotin-conjugated anti-rat IgG, respectively. (B) Schematic illustration of DaMab-8 epitope. (C) Schematic illustration of DaMab-2 and DaMab-8 epitopes. Red amino acids, strong reaction with DaMab-8; brown amino acids, weak reaction with DaMab-8. PVDF, polyvinylidene difluoride. 
deletion mutants were detected by an anti-PA tag mAb, NZ1 (Fig. 2A), indicating that the N-terminus of the DaMab-8epitope exists between amino acids 601 and 621 (Fig. 2B). Western blotting of the additional deletion mutants of DGK $\alpha$ - that is, dN606, dN611, and dN616-demonstrated that DaMab-8 detected dN606 and dN611, but not dN616, although all deletion mutants were detected by an anti-PA tag mAb, NZ-1 (Fig. 3A), thereby indicating that the Nterminus of the DaMab-8-epitope exists between amino acids 611 and 616 (Fig. 3B).

Accordingly, we produced the following $26 \mathrm{DGK} \alpha$ point mutants: M605A, H606A, G607A, G608A, S609A, N610A, L611A, W612A, G613A, D614A, T615A, R616A, R617A, P618A, H619A, G620A, D621A, I622A, Y623A, G624A, I625A, N626A, Q627A, A628G, L629A, and G630A. Western blotting demonstrated that the anti-PA tag mAb, NZ-1, detected all point mutants (Fig. 4A). In contrast, DaMab-8 strongly detected M605A, H606A, G607A, G608A, S609A, T615A, R616A, R617A, P618A, G620A, D621A, I622A, I625A, N626A, Q627A, A628G, L629A, and G630A; weakly detected N610A, W612A, and D614A; and did not detect mutants L611A, G613A, H619A, Y623A, and G624A (Fig. 4A). DaMab-8 epitopes are summarized in Figure 4B.

In conclusion, Asn610, Leu611, Trp612, Gly613, Asp614, His619, Tyr623, and Gly624 are important for facilitating DaMab- 8 binding to the DGK $\alpha$ protein. This epitope exists in the catalytic domain of DGK $\alpha$, whereas DaMab-2 binds to Zn-finger (Fig. 4C). DaMab-8 could be advantageous for immunohistochemical analyses toward clarifying the distribution of DGK $\alpha$-expressing $\mathrm{T}$ cells in every pathophysiological tissue. Furthermore, these findings could be applied for the production of more functional anti-DGK $\alpha \mathrm{mAbs}$.

\section{Acknowledgments}

We thank Shunsuke Itai, Miyuki Yanaka, Kayo Hisamatsu, Saori Handa, Yoshimi Nakamura, and Maki Takahashi for their excellent technical assistance, and Shinji Yamada for his helpful discussion. This research was supported, in part, by AMED under Grant Numbers: JP18am0101te078 (Y.K.), JP18am0301010 (Y.K.), and JP18ae0101028 (Y.K.), and by JSPS KAKENHI Grant Numbers 17K07299 (M.K.K.) and 16K10748 (Y.K.).

\section{Author Disclosure Statement}

Y.K. received research funding from Ono Pharmaceutical Co., Ltd. The other authors have no conflict of interest.

\section{References}

1. Topham MK, and Epand RM: Mammalian diacylglycerol kinases: molecular interactions and biological functions of selected isoforms. Biochim Biophys Acta 2009;1790:416424.
2. Goto K, Hozumi Y, Nakano T, Saino SS, and Kondo H: Cell biology and pathophysiology of the diacylglycerol kinase family: morphological aspects in tissues and organs. Int Rev Cytol 2007;264:25-63.

3. Sakane F, Imai S, Kai M, Yasuda S, and Kanoh H: Diacylglycerol kinases: why so many of them? Biochim Biophys Acta 2007;1771:793-806.

4. Jones DR, D'Santos CS, Merida I, and Divecha N: T lymphocyte nuclear diacylglycerol is derived from both de novo synthesis and phosphoinositide hydrolysis. Int $\mathrm{J}$ Biochem Cell Biol 2002;34:158-168.

5. Olenchock BA, Guo R, Carpenter JH, Jordan M, Topham MK, Koretzky GA, and Zhong XP: Disruption of diacylglycerol metabolism impairs the induction of $\mathrm{T}$ cell anergy. Nat Immunol 2006;7:1174-1181.

6. Foell J, Hewes B, and Mittler RS: T cell costimulatory and inhibitory receptors as therapeutic targets for inducing antitumor immunity. Curr Cancer Drug Targets 2007;7:55-70.

7. Nakano T, Ogasawara S, Tanaka T, Hozumi Y, Mizuno S, Satoh E, Sakane F, Okada N, Taketomi A, Honma R, Nakamura T, Saidoh N, Yanaka M, Itai S, Handa S, Chang YW, Yamada S, Kaneko MK, Kato Y, and Goto K: DaMab-2: anti-human DGKalpha monoclonal antibody for immunocytochemistry. Monoclon Antib Immunodiagn Immunother 2017;36:181-184.

8. Sano M, Kaneko MK, and Kato Y: Epitope mapping of antidiacylglycerol kinase alpha monoclonal antibody DaMab-2. Monoclon Antib Immunodiagn Immunother 2019;38:8-11.

9. Schaap D, de Widt J, van der Wal J, Vandekerckhove J, van Damme J, Gussow D, Ploegh HL, van Blitterswijk WJ, and van der Bend RL: Purification, cDNA-cloning and expression of human diacylglycerol kinase. FEBS Lett 1990; 275:151-158.

10. Fujii Y, Kaneko M, Neyazaki M, Nogi T, Kato Y, and Takagi J: PA tag: a versatile protein tagging system using a super high affinity antibody against a dodecapeptide derived from human podoplanin. Protein Expr Purif 2014;95: 240-247.

11. Wang Y, Zhang Q, Ma Q, Zhang Y, Li Z, and Wang C: DGKalpha DNA vaccine relieves airway allergic inflammation in asthma model possibly via induction of $\mathrm{T}$ cell anergy. Int J Clin Exp Pathol 2013;6:2404-2411.

Address correspondence to: Yukinari Kato New Industry Creation Hatchery Center Tohoku University 2-1, Seiryo-machi, Aoba-ku Sendai 980-8575 Japan

E-mail: yukinarikato@med.tohoku.ac.jp Received: January 30, 2019 Accepted: March 22, 2019 